\title{
LINC00689 promotes prostate cancer progression via regulating miR-496/CTNNB1 to activate Wnt pathway
}

Liwei Meng ${ }^{1}$, Zhonghai Li ${ }^{2}$, Ye Chen ${ }^{2}$, Degian Liu² and Zhaoxu Liu ${ }^{1} 3^{*}$ (1)

\section{Abstract}

Background: Accumulating evidence has proved the significant influence of long non-coding RNAs (IncRNAs) in cancer formation and development, including PCa.

Methods: The role of LINC00689 in PCa was confirmed by RT-qPCR, MTT, colony formation, flow cytometry, western blot and transwell assays. Besides, the binding ability between LINC00689 and miR-496 was validated by using luciferase reporter assay. Then RT-qPCR, RIP and luciferase reporter and western blot assays were employed to verify the interactions among LINC00689, miR-496 and CTNNB1. Furthermore, the rescuing role of CTNNB1 in Wnt pathway was proved by RT-qPCR, TOP/FOP Flash and western blot assays.

Results: LINC00689 was upregulated in PCa tissues and cells as well as at the terminal stage. Further, knock down of LINC00689 repressed PCa cell proliferation, migration and invasion, and initiated PCa cell apoptosis. Additionally, miR-496 inhibitor and pcDNA3.1/CTNNB1 could neutralize the prohibitive effects of LINC00689 silencing on cell proliferation, migration and invasion, meanwhile, could offset the encouraging role of knocking down LINC00689 in cell apoptosis. Moreover, CTNNB1 upregulation exerted redemptive function in Wnt pathway inhibited by LINC00689 depletion.

Conclusions: To sum up, LINC00689 promotes PCa progression via regulating miR-496/CTNNB1 to activate Wnt

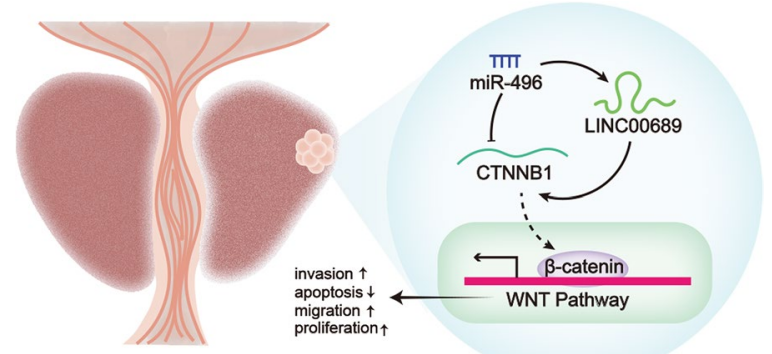

pathway, which may contribute to research about new targets for PCa treatment.

Keywords: Prostate cancer, LINC00689, miR-496, CTNNB1, Wnt pathway

*Correspondence: Izx4kk@163.com

1 Department of Urology, Qilu Hospital of Shandong University, No.107 Culture Road, Jinan 250000, Shandong, China

Full list of author information is available at the end of the article

\section{Background}

Prostate cancer $(\mathrm{PCa})$ is identified as a type of the most common male malignancies in the world, with an

(c) The Author(s) 2020. This article is licensed under a Creative Commons Attribution 4.0 International License, which permits use, sharing, adaptation, distribution and reproduction in any medium or format, as long as you give appropriate credit to the original author(s) and the source, provide a link to the Creative Commons licence, and indicate if changes were made. The images or other third party material in this article are included in the article's Creative Commons licence, unless indicated otherwise in a credit line to the material. If material is not included in the article's Creative Commons licence and your intended use is not permitted by statutory regulation or exceeds the permitted use, you will need to obtain permission directly from the copyright holder. To view a copy of this licence, visit http://creativeco mmons.org/licenses/by/4.0/. The Creative Commons Public Domain Dedication waiver (http://creativecommons.org/publicdomain/ zero/1.0/) applies to the data made available in this article, unless otherwise stated in a credit line to the data. 
increasing incidence and mortality in recent years [13]. The epidemiological survey shows that in the past 10 years, the developed degree of a country is negatively correlated with the death rate of PCa patients, that is, the more backward the country, the higher the fatality rate of $\mathrm{PCa}$ [4]. Considering the clinical value of $\mathrm{PCa}$, the occurrence of tumors and effective treatment methods need to be studied in-depth.

Long non-coding RNAs (lncRNAs) were initially identified as the "garbage" of genomic transcription. Nevertheless, recent researches have elucidated that lncRNAs are involved in regulating molecular processes, such as $\mathrm{X}$-chromosome silencing, gene imprinting, chromatin modification, transcriptional activation, transcriptional interference, and intra-nuclear transport, which begin to attract widespread attention [5-10]. During the development of PCa, lncRNAs play an important regulatory role. For instance, androgen-induced lncRNA SOCS2-AS1 facilitates PCa cell proliferation and prohibits apoptosis [11]. LncRNA MALAT-1 is recognized as a newly-found possible therapy target for $\mathrm{PCa}$ with castration resistance [12]. Low BDNF-AS expression is related to the unsatisfactory prognosis of $\mathrm{PCa}$ patients [13]. Further, LINC00689 has recently drawn attention when studying its role in cancer progression. However, the number of the concerned research is limited [14]. Therefore, the regulation mechanism of LINC00689 in PCa remains a novel topic of concern in this study.

In our research, LINC00689 promotes cell proliferation, migration, invasion as well as suppresses cell apoptosis via regulating miR-496/CTNNB1 to activate Wnt pathway, which may contribute to find a fresh target for PCa treatment.

\section{Methods}

\section{Tissue samples}

80 patients chosen from Affiliated Hospital of Jining Medical University were included in this research. None of the patients underwent chemo- or radiation therapy. Following surgical resection, tumor tissues were quickly frozen in liquid nitrogen and subsequently stored at $-80{ }^{\circ} \mathrm{C}$ for further use. The present research was favored by the Ethics Committee of Affiliated Hospital of Jining Medical University. Informed consent was attained from all the patients.

\section{Cell culture}

Normal prostate epithelial cell (RWPE1) and PCa cells (DU145, LNCaP, PC-3 and C42B) were bought from American Type Culture Collection (ATCC; Manassas, VA, USA). Cells were cultured in line with previous description [15]. They were cultured with 10\% FBS and
1\% antibiotics in DMEM (Gibco, Rockville, MD, USA). In order to activate the $\mathrm{Wnt} / \beta$-catenin signaling pathway, DU145 cells were treated with lithium chloride ( $\mathrm{LiCl}$; Sigma-Aldrich, St. Louis, MO, USA) for $24 \mathrm{~h}$.

\section{Cell transfection}

Specific shRNAs against LINC00689 (sh-LINC00689\#1 and sh-LINC00689\#2) and their corresponding NC (sh$\mathrm{NC}$ ), as well as the pcDNA3.1 vector containing the whole sequence of LINC00689 or CTNNB1 and the empty vector, were attained from Genechem (Shanghai, China). The miR-496 mimics, miR-496 inhibitors, NC mimics and NC inhibitors were constructed by GenePharma (Shanghai, China). By use of Lipofectamine 3000 (Invitrogen, Carlsbad, CA, USA), plasmids mentioned were individually transfected into DU145 or LNCaP cells in 24-well plates for $48 \mathrm{~h}$. Sequences for shRNAs were listed as follows: sh-NC: CCGG TCTTGCGTC GTCTGTCTATAC CTCGAG GTATAGACAGACGAC GCAAGA TTTTTG; sh-LINC00689\#1: CCGG GCG TCTTTCCTTCTGTTAAGC CTCGAG GCTTAACAG AAGGAAAGACGC TTTTTG; CCGG GCTTCTGCT TTCCTGAAATTC CTCGAG GAATTTCAGGAA AGCAGAAGC TTTTTG. Plasmids' sequences were shown as follows: NC mimics: gcugcauaucaguaucuacaug; miR-496 mimics: ugaguauuacauggccaaucuc; NC inhibitors: uagacaggcauguaauguacuc; miR-496 inhibitors: gagauuggccauguaauacuca.

\section{RT-qPCR (real-time quantitative polymerase chain reaction)}

Total RNAs were extracted from tissues or cells by utilizing TRIzol reagents (Invitrogen), and then reverse-transcribed into cDNA in line with the protocol of a reverse transcriptase kit (Takara, Dalian, China). Next, RT-qPCR was undertaken with TB Green Premix ExTaq II (Takara) at the Applied Biosystems 7500 Real Time PCR system (Applied Biosystems, Foster City, CA, USA). Relative gene expression was normalized to GAPDH or U6. Quantification of relative gene expression was conducted via comparative $2^{-\Delta \Delta \mathrm{Ct}}$ approach. Assay was undertaken in at least triplicate. Primer sequences were listed as follows: LINC00689 (F): AGTTGGTACAGGGAGGGGTT; LINC00689 (R): GTCCCTCTTGGTGGAGTTGG; miR496 (R): tgagtattacatggccaatctc; miR-496 (F): GCCGAG tgagtattacatggcc; GAPDH (F): GGAGCGAGATCCCTC CAAAAT; GAPDH (R): GGCTGTTGTCATACTTCT CATGG; U6 (F): CTCGCTTCGGCAGCACA; U6 (R): AACGCTTCACGAATTTGCGT; CTNNB1 (F): ACG GAGGAAGGTCTGAGGAG; CTNNB1 (R): AGCCGC TTTTCTGTCTGGT. 


\section{MTT (methyl thiazolyl tetrazolium) assay}

Cell viability was explored with the MTT assay kit (Invitrogen). In short, transfected DU145 or LNCaP cells were seeded at the density of $1 \times 10^{3}$ cells per well to 96-well plates, washed in phosphate-buffered saline (PBS; Sigma-Aldrich) and sequentially incubated for $4 \mathrm{~h}$ using MTT solution. Upon incubation, medium was exchanged into DMSO and cells were incubated for $10 \mathrm{~min}$. OD490 $\mathrm{nm}$ value was read with a microplate reader. Assay was implemented in at least triplicate.

\section{Colony formation assay}

Transfected DU145 or LNCaP cells were harvested after $48 \mathrm{~h}$ and added onto 6-well plates. 14 days later, cells were fixed by $4 \%$ formaldehyde (Sigma-Aldrich) for $30 \mathrm{~min}$, stained for $5 \mathrm{~min}$ in $0.1 \%$ crystal violet solution (Sigma-Aldrich). Colonies were photographed by a camera (Canon, Japan) and the visible colonies containing over 50 cells were counted, manually. Assay was performed in at least triplicate.

\section{Cell apoptosis assay}

The Annexin V-FITC Apoptosis Detection Kit (BD, San Diego, CA, USA) was employed to examine cell apoptosis. Transfected DU145 or LNCaP cells were washed in cold PBS, followed by re-suspended in 6-well plates with $200 \mu \mathrm{L}$ binding buffer (Thermo Fisher Scientific, Waltham, MA, USA) adding fluorescein isothiocyanate (FITC)-labeled annexin V and PI (Sigma-Aldrich). Upon incubation without light for $30 \mathrm{~min}, 300 \mu \mathrm{L}$ binding buffer was supplemented. Flow cytometry (Beckman Coulter, Brea, CA, USA) was conducted for analysis. Assay was conducted in at least triplicate.

\section{Caspase- 3 activity assay}

Transfected DU145 or LNCaP cells were lysed in lysis buffer (Invitrogen) and cell protein extracts were added in Ac-DEVD-AMC (Beyotime, Haimen, China) and sequentially incubated for $1 \mathrm{~h}$. The caspase- 3 activity kit was procured from Beyotime and used as required. Suspension was then placed in the influorescence spectrometer (Promega, Madison, WI, USA) so as to analyze influorescent intensity at $405 \mathrm{~nm}$. This assay was conducted in at least triplicate.

\section{Transwell assay}

On the one hand, transfected DU145 or LNCaP cells $\left(1 \times 10^{5}\right)$ were added in the upper uncoated (for migration) or $0.5 \mathrm{~mm}$ of standard Matrigel (Cat\#356234, BD)-coated (for invasion) transwell chambers $(8 \mu \mathrm{m}$ pore size; $\mathrm{BD}$ ) with serum-free medium. On the other hand, culture medium with $10 \%$ fetal bovine serum
(FBS; PAN-Biotech, Adenbach, Bagolia, Germany) was loaded to the lower wells. $24 \mathrm{~h}$ later, the non-migratory or non-invasive cells were wiped out. The filters were fixed, and stained by crystal violet solution. Five randomly picked fields were counted per chamber using the inverted microscope $(4 \times$ objective lens). This assay was performed in at least triplicate.

\section{Bioinformatics analysis}

MiRNAs that can bind with LINC00689 were predicted by starBase v3.0 (http://starbase.sysu.edu.cn/). The downstream target mRNAs of miR-496 were predicted from starBase v3.0 (http://starbase.sysu.edu.cn/) with five program numbers.

\section{Luciferase reporter assay}

LINC00689 or CTNNB1 3'UTR fragments covering wild-type and mutant miR-496 binding sites were inserted into the pmirGLO dual-luciferase plasmid (Promega), and pmirGLO-LINC00689-WT/Mut or pmirGLO-CTNNB1 $3^{\prime}$ UTR-WT/Mut was thus formed. The pmirGLO-LINC00689-WT/Mut was co-transfected into DU145 or LNCaP cells with miR-496 mimics or NC mimics. The pmirGLO-CTNNB1 $3^{\prime}$ UTR-WT/Mut was co-transfected into DU145 or LNCaP cells with miR-496 mimics or miR-496 mimics + pcDNA3.1/LINC00689 or NC mimics. For detecting luciferase activities, dual luciferase reporter assay system (Promega) was applied. This assay was undertaken in at least triplicate.

\section{RNA immunoprecipitation (RIP)}

The RIP assay was implemented via a Magna RIP RNA Binding Protein Immunoprecipitation Kit (Bersinbio, Guangzhou, China). DU145 or LNCaP cells were lysed adopting RIP lysis buffer. Cell lysates were then divided into two equivalent parts for incubating with either antiAgo2 antibody (ab32381, Abcam, Cambridge, MA, USA) or non-specific anti-IgG antibody (ab190475, Abcam). Magnetic beads (Invitrogen) were supplemented to cell lysates and incubation was continued for $1 \mathrm{~h}$, after which were incubated with Proteinase K (Absin, Shanghai, China) for $1 \mathrm{~h}$ at $55{ }^{\circ} \mathrm{C}$. Detection of the enriched RNA was subjected to RT-qPCR. Assay was undertaken in at least triplicate.

\section{Western blot}

Transfected DU145 or LNCaP cells with or without $\mathrm{LiCl}$ treatment were lysed in RIPA buffer (Thermo Fisher Scientific) with additional blends of protease inhibitors (Roche, Mannheim, Germany). Lysate was collected and subjected to SDS-PAGE (Bio-Rad, Hercules, CA, USA), after which were transferred onto PVDF membranes 
(Bio-Rad). Upon incubation with Blocking One reagent (Nacalai Tesque, Kyoto, Japan), membranes were blotted by primary antibodies against CTNNB1 (SAB2701829, Sigma-Aldrich), $\beta$-catenin (ab32572, Abcam), CCND1 (SAB1405510, Sigma-Aldrich), CDK2 (ab32147, Abcam), c-MYC (ab32072, Abcam), and GAPDH (ab245356, Abcam) and then by HRP-labeled secondary antibodies. The signals were monitored after washing using the ECL Western blot kit (Thermo Fisher Scientific). Assay was undertaken in at least triplicate.

\section{TOP/FOP flash}

DU145 cells were co-transfected with sh-LINC00689\#1 or sh-LINC00689\#1+ pcDNA3.1/CTNNB1 or shLINC00689\# $1+\mathrm{LiCl}$ or sh-NC and TOP Flash or FOP Flash plasmids (Upstate Biotechnology, Lake Placid, NY, USA). For detecting luciferase activities, dual luciferase reporter assay system (Promega) was applied. Assay was undertaken in at least triplicate.
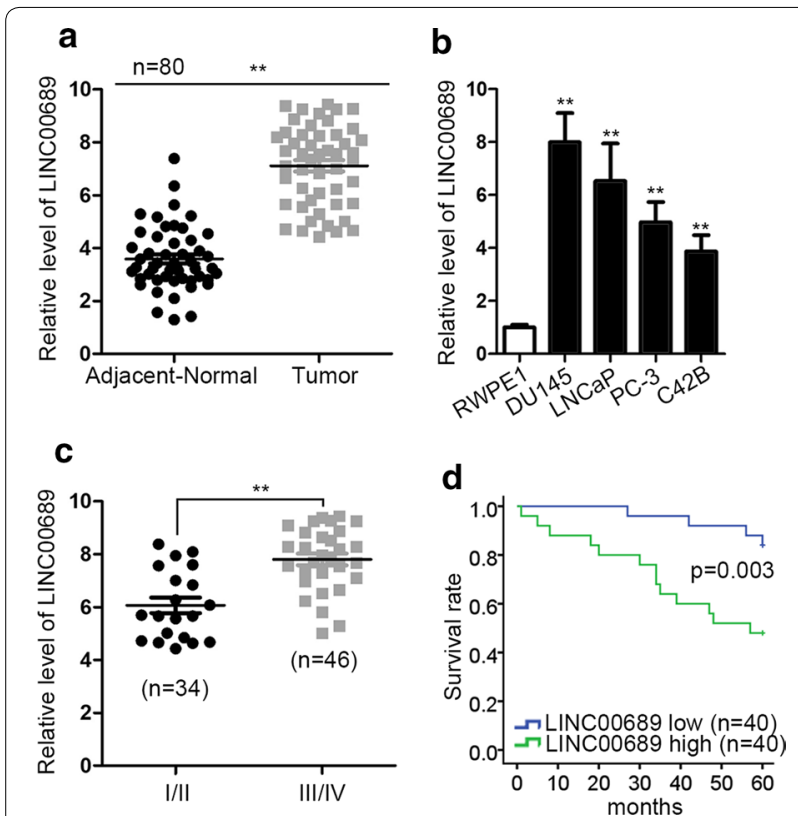

Fig. 1 LINC00689 is upregulated in prostate cancer tissues and cells. a The expression of LINC00689 in adjacent-normal $(n=80)$ and tumor $(n=80)$ tissues was detected by RT-qPCR. $\mathbf{b}$ The expression of LINC00689 in normal prostate epithelial cell (RWPE1) and PCa cells (DU145, LNCaP, PC-3 and C42B) were detected by RT-qPCR. c Relative LINC00689 expression in different clinical stages (early I/II and advanced III/IV) was measured by RT-qPCR. d Kaplan-Meier curve and the log-rank test showed that the correlation between high or low LINC00689 expression and overall survival in patients with PCa. Error bars represent the mean $\pm S D$ of at least three independent experiments. ${ }^{* *} P<0.01$

\section{Statistical analysis}

All assays were undertaken in triplicate. $\mathrm{P}<0.05$ was considered statistically significant. For analyzing the experimental data which were expressed as mean \pm SD, SPSS 17.0 software (IBM, Armonk, NY, USA) was used. Student's t-test was conducted for comparing two groups, and one-way ANOVA was for comparing multiple groups. Survival rate was assayed with Kaplan-Meier approach, and difference was analyzed by a log-rank test.

\section{Results}

\section{LINC00689 is upregulated in prostate cancer tissues} and cells

To verify the role of LINC00689 in PCa, RT-qPCR assessed that the expression of LINC00689 was much higher in PCa tissues than that in adjacent normal tissues (Fig. 1a). Meanwhile, LINC00689 expressed much higher in PCa cells (DU145, LNCaP, PC-3 and C42B) than control cells. Besides, DU145 and LNCaP contained the most expression of LINC00689 (Fig. 1b). Further, the patients with $\mathrm{PCa}$ at the advanced stage (III-IV) possessed much more LINC00689 expression than the patients at an early stage (I-II) (Fig. 1c). In addition, Kaplan-Meier curve in Fig. 1d depicted that high LINC00689 expression was closely associated with short overall survival time of $\mathrm{PCa}$ patients, indicating the unsatisfactory prognosis of $\mathrm{PCa}$ patients was induced by high LINC00689 level. Above data indicated that LINC00689 serves as a potential oncogene in PCa.

\section{Knock down of LINC00689 restrains prostate cancer progression}

For comprehending the regulation mechanism of LINC00689 in PCa, firstly, the knockdown efficiency of LINC00689 was detected in DU145 and LNCaP cells using sh-LINC00689\#1/\#2. The knockdown efficiency of sh-LINC00689\#1 was better (Fig. 2a). Then followed by several functional assays, MTT assay validated that silencing of LINC00689 suppressed cell proliferation in DU145 and LNCaP cells. Hereinto, the effectiveness of LINC00689\#1 was more evident (Fig. 2b). And colony formation assay confirmed the obstructive role of LINC00689 depletion in cell proliferation based on the decline in colony number of DU145 and LNCaP cells (Fig. 2c). With regard to cell apoptosis, flow cytometry detected that LINC00689 downregulation induced about two folds of increase in cell apoptosis rate in DU145 and LNCaP cells (Fig. 2d). Western blot assay measured the upregulated protein level of cleaved caspase 3 was caused by LINC00689 knockdown (Fig. 2e and Additional file 1a). Similarly, Caspase- 3 test illustrated that absence of LINC00689 motivated cell apoptosis (Fig. 2f). Subsequently, transwell assay determined that shortage of 

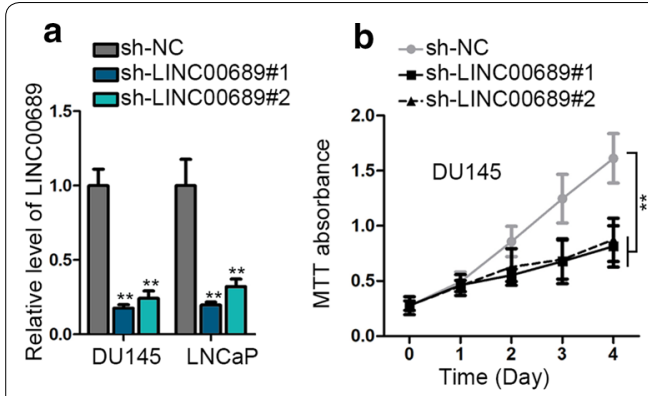
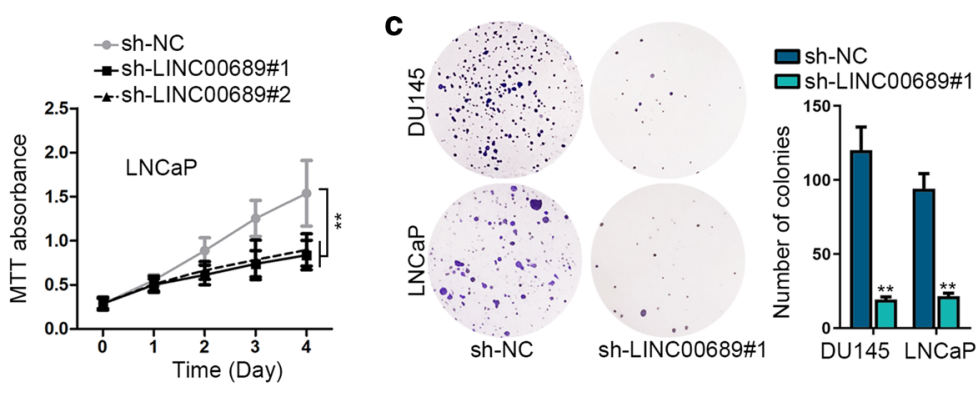
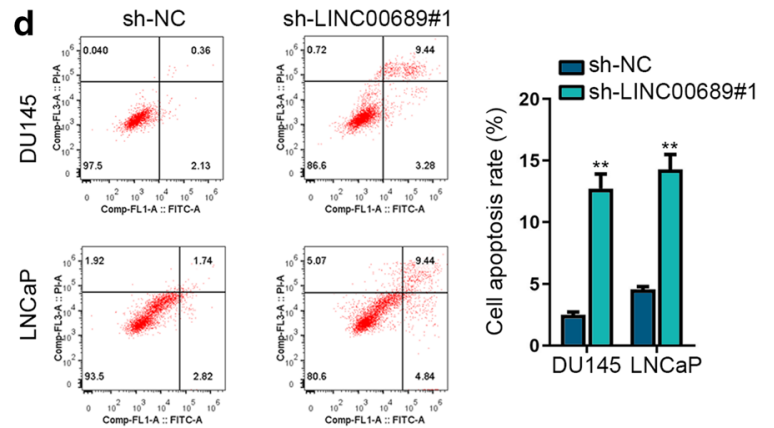

e

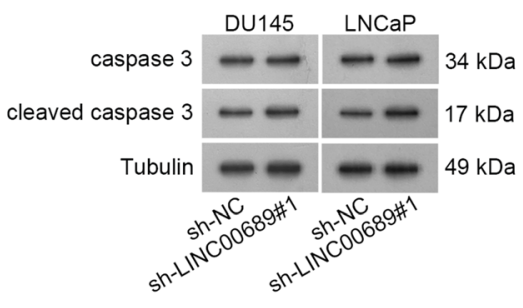

f

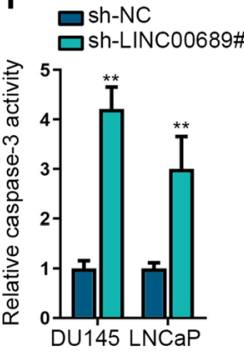

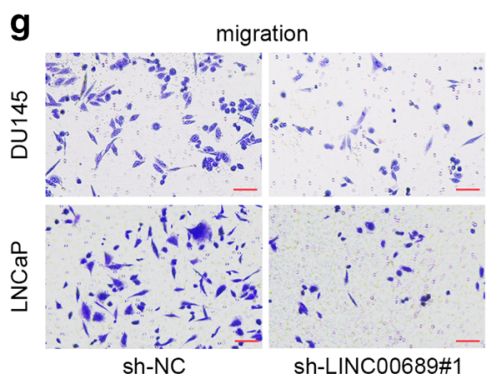
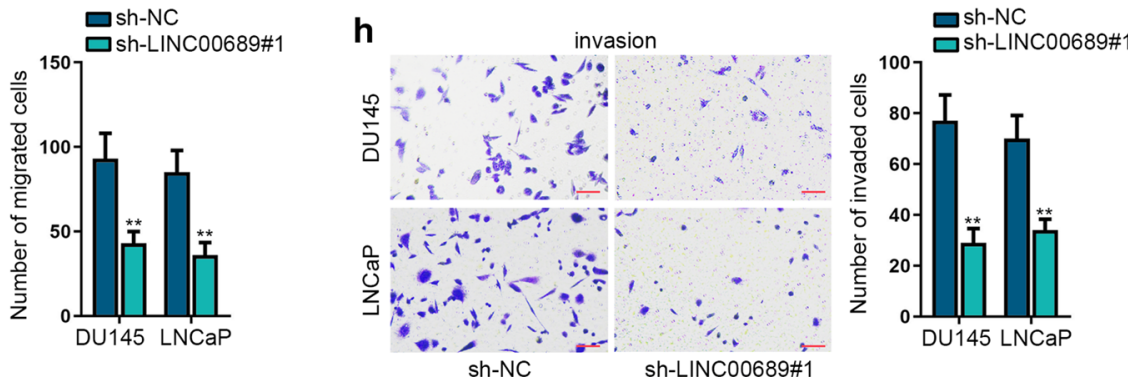

Fig. 2 Knock down of LINC00689 restrains prostate cancer progression. a The knockdown efficiency of LINC00689 was detected in DU145 and LNCaP cells after $48 \mathrm{~h}$ of transfection with sh-LINC00689\#1/\#2. b, c MTT (detected after incubated for indicated time) and colony formation (measured after incubated for 14 days) assays measured the effects of knocking down LINC00689\#1/\#2 on DU145 and LNCaP cell proliferation. $\mathbf{d}$ Flow cytometry analysis measured the apoptosis in LINC00689 silenced DU145 and LNCaP cells. e Western blot assay examined that protein level of cleaved caspase 3 in sh-LINC00689\#1 transfected DU145 and LNCaP cells. GAPDH was an internal control. $\mathbf{f}$ Caspase-3 test displayed the caspase-3 activity in response to LINC00689 downregulation in DU145 and LNCaP cells. $\mathbf{g}, \mathbf{h}$ Transwell assay determined the effects of LINC00689 silencing on DU145 and LNCaP cell migration and invasion after incubated for $24 \mathrm{~h}$. Images were captured by using the inverted microscope $(4 \times$ objective lens) (scale bar $=100 \mu \mathrm{m}$ ). Error bars represent the mean \pm SD of at least three independent experiments. ${ }^{* *} \mathrm{P}<0.01$

LINC00689 prohibited cell migration and invasion referring with the reduced number of migrated and invaded cells (Fig. 2g, h). Above all, knockdown of LINC00689 restrains PCa progression.

\section{LINC00689 sponges with miR-496 in prostate cancer}

For the purpose of finding the microRNA (miRNA) sponged by LINC00689, we searched from starBase v3.0. Among all candidate miRNAs, miR-496 has been reported to be downregulated in PCa samples [16]. As shown in Fig. 3a and Additional file 2, the binding sites of LINC00689 and miR-496 predicted by starBase were displayed. Luciferase reporter assay confirmed the binding relation between LINC00689 and miR-496. Luciferase activity of LINC00689-WT was reduced in miR-496 mimics transfected cells (Fig. 3b). RT-qPCR measured that sh-LINC00689 increased the expression of miR496 in DU145 and LNCaP cells (Fig. 3c). Moreover, the expression of miR-496 in normal prostate epithelial cell (RWPE1) and PCa cells (DU145, LNCaP, PC-3 and C42B) were detected, and miR-496 was downregulated in PCa cells in comparison with that in RWPE1 cell (Fig. 3d). The knockdown efficiency of miR-496 was also measured in miR-496 inhibitor transfected cells (Fig. 3e). Finally, the 


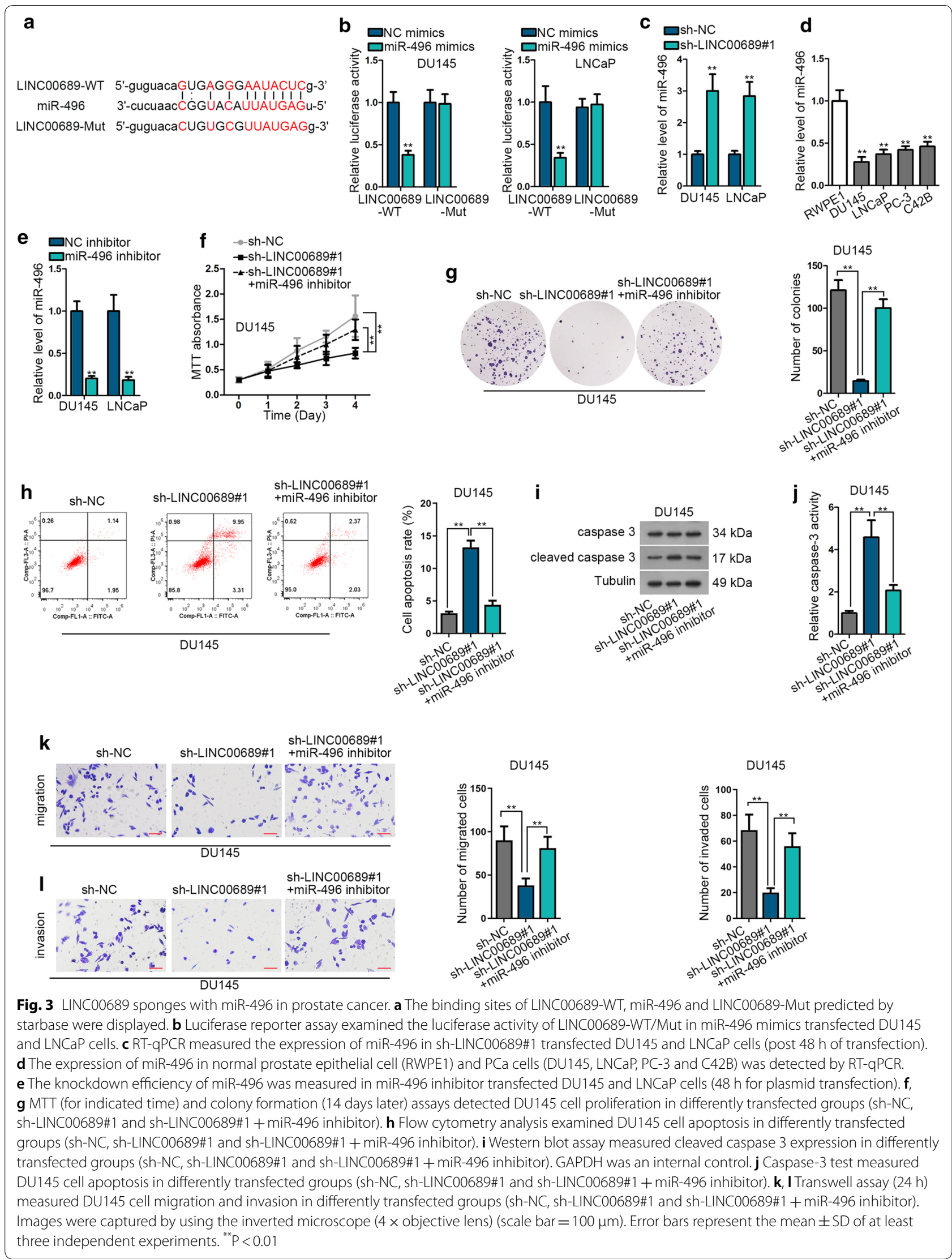



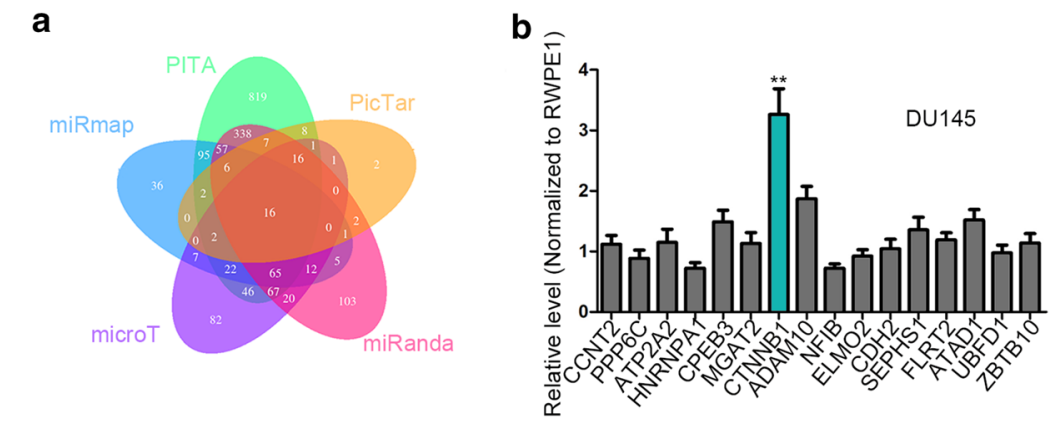

C

$\begin{array}{cc}\text { CTNNB1 3'UTR-WT } & \text { 5'-agcuUUUGCAACUUAAUACUCa-3' } \\ 111111111111 \\ \text { miR-496 } & \text { 3'-CUCUAACCGGUACAUUAUGAGU-5' }\end{array}$ CTNNB1 3'UtR-Mut 5'-agcuAAUCGAUCUAUUAUGAGa-3'
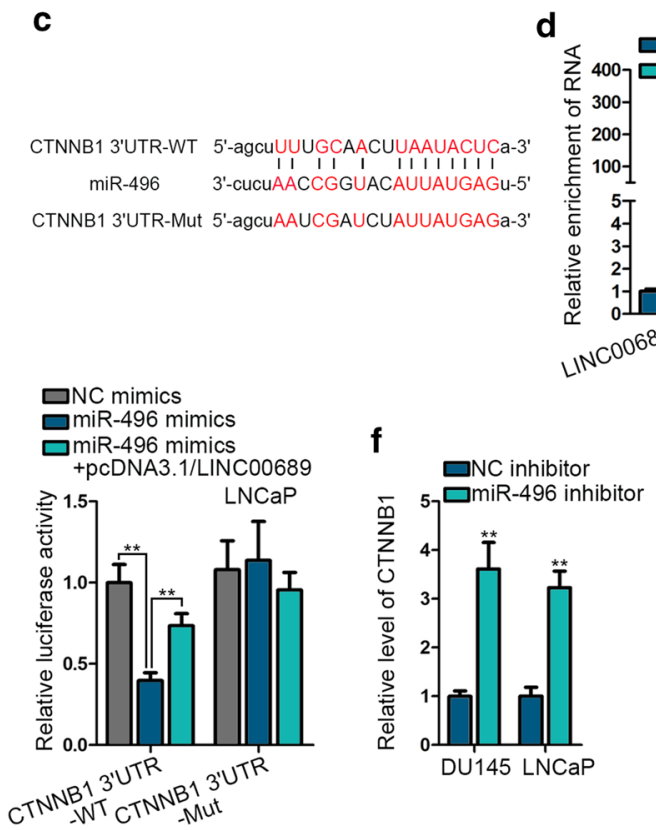
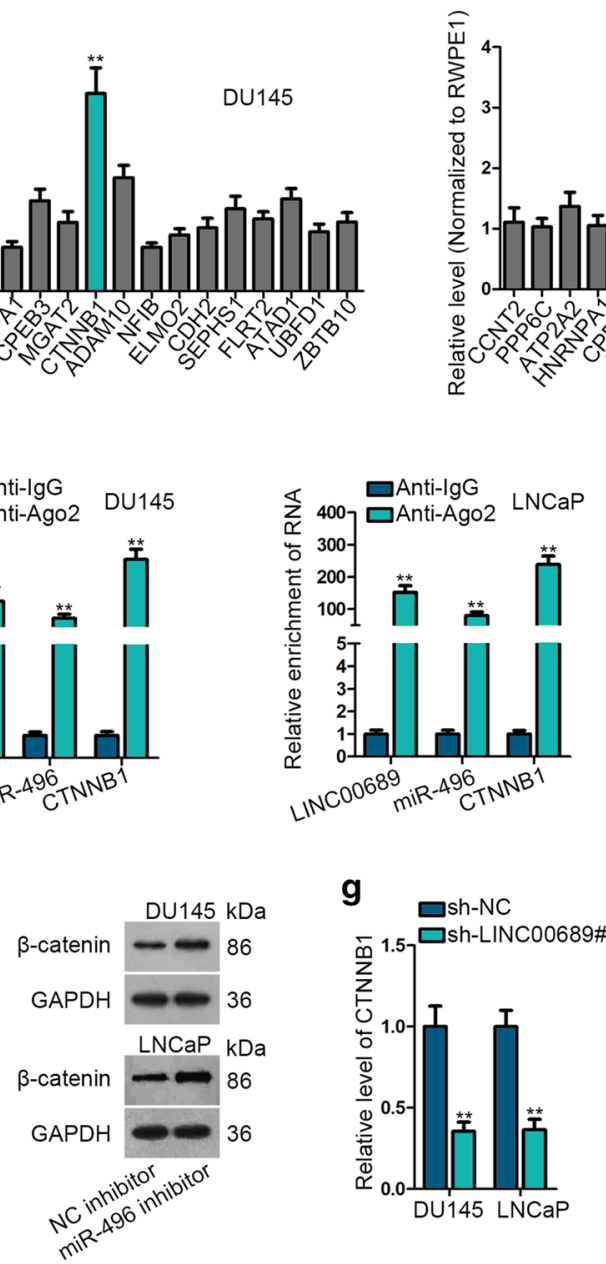

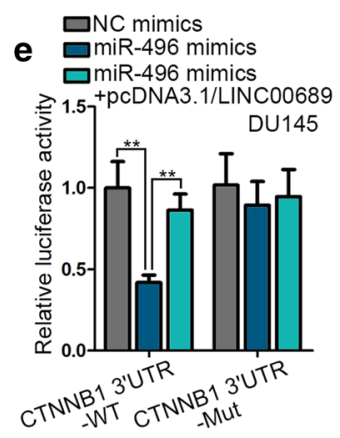

Fig. 4 MiR-496 targets to CTNNB1 in prostate cancer. a Venn diagram exhibited 16 qualified messenger RNAs (mRNAs) collected by using starbase. $\mathbf{b}$ The expression of 16 mRNAs in normal prostate epithelial cell (RWPE1) and PCa cells (DU145 and LNCaP) was detected by RT-qPCR. c The conjectured binding sites between miR-496 and CTNNB1 were showed. $\mathbf{d}$ RIP assay detected the enrichment of LINC00689, miR-496 and CTNNB1 in Anti-lgG or Anti-Ago of DU145 and LNCaP cells. e Luciferase reporter assay examined the luciferase activity of CTNNB1 $3^{\prime} U$ TTR-WT/Mut in transfected DU145 and LNCaP cells (NC mimics, miR-496 mimics and miR-496 mimics + pcDNA3.1/LINC00689). $\mathbf{f}, \mathbf{g}$ RNA or protein level of CTNNB1 or $\beta$-catenin was measured respectively by RT-qPCR and western blotting in miR-496 inhibitor and sh-LINC00689\#1 transfected DU145 and LNCaP cells (48 $\mathrm{h}$ for plasmid transfection). GAPDH was an internal control. Error bars represent the mean \pm SD of at least three independent experiments. ${ }^{* *} \mathrm{P}<0.01$

rescuing function of miR-496 inhibitor in sh-LINC00689 transfected cells was elucidated. MiR-496 inhibition canceled out restraining effects of sh-LINC00689 on cell proliferation (Fig. 3f, g). As for PCa cell apoptosis, in follow cytometry assay, when downregulating LINC00689, the DU145 cell apoptosis rate was three times as much as the control group. And cell apoptosis rate exhibited two folds of decrease in sh-LINC00689\#1+miR-496 inhibitor group compared with sh-LINC00689\#1 group (Fig. 3h). In western blot analysis and caspase-3 activity detection, miR-496 inhibitor counteracted the encouraging effects of LINC00689 depletion on cleaved caspase 3 protein level and Caspase-3 activity (Fig. 3i, $\mathbf{j}$ and Additional file 1b). Similarly, the depressing function caused by LINC00689 insufficiency on cell migration and invasion was reversed by inhibition of miR-496 (Fig. 3k, l). In a word, miR-496 is sponged by LINC00689, and miR496 inhibitor could partly rescue the suppressive role of LINC00689 knockdown in PCa cellular activities.

\section{MiR-496 targets to CTNNB1 in prostate cancer}

Based on the above results, we needed to figure out the target gene of miR-496 to complete competing endogenous RNA (ceRNA) regulation mechanism of 
LINC00689 in PCa. By utilizing starBase, 16 qualified messenger RNAs (mRNAs) were plotted in Venn diagram (Fig. 4a). The expression of the indicated mRNAs in normal prostate epithelial cell (RWPE1) and PCa cells (DU145 and LNCaP) was detected by RT-qPCR. CTNNB1 was prominently upregulated in DU145 and LNCaP cells compared with normal prostate epithelial cell (RWPE1) (Fig. 4b). In hence, CTNNB1 was chose to perform the following assays. The predicted binding site between miR-496 and CTNNB1 was showed in Fig. 4c.
And RIP assay validated that LINC00689, miR-496 and CTNNB1 coexisted in RISC (RNA-induced silencing complex) (Fig. 4d), indirectly confirming the interactions among these three genes in PCa cells. And luciferase reporter assay verified the binding facts between miR496 and CTNNB1. Besides, pcDNA3.1/LINC00689 remedied the blocking effect of miR-496 mimics on luciferase activity of CTNNB1 3'UTR-WT (Fig. 4e), suggesting that LINC00689 could upregulate CTNNB1 via sponging miR-496. Moreover, the mRNA expression of CTNNB1

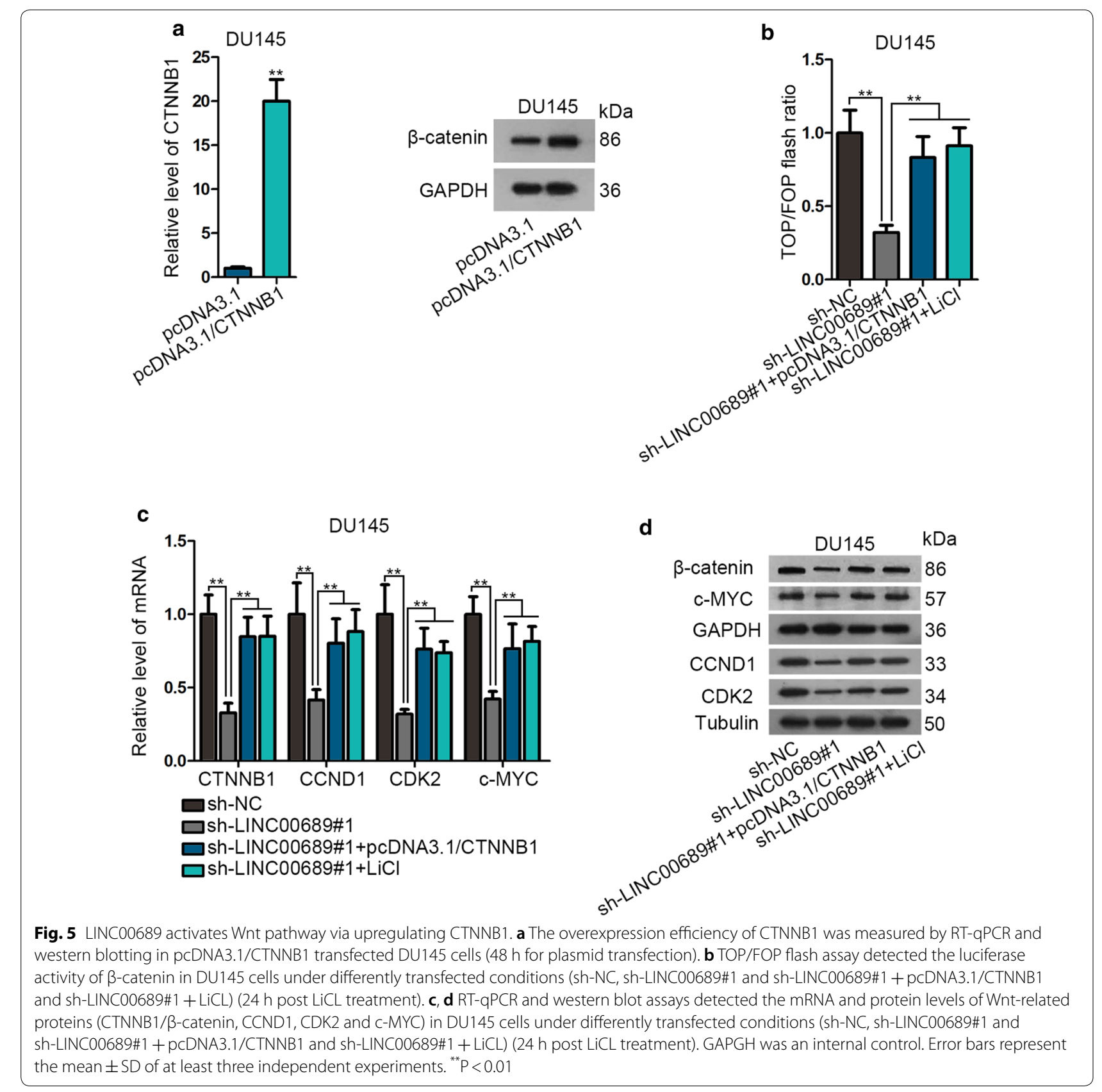


and protein level of $\beta$-catenin (protein form of CTNNB1) were measured in miR-496 inhibitor and sh-LINC00689 transfected cells, respectively. And obviously, the miR496 inhibitor stimulated the mRNA and protein levels of CTNNB1, however, sh-LINC00689 led to completely opposite results (Fig. 4f, g). On the whole, CTNNB1 is targeted by miR-496, and the expression of CTNNB1 is negatively/positively regulated by miR-496/LINC00689 in PCa cells.

\section{LINC00689 activates Wnt pathway via upregulating CTNNB1}

Wnt pathway is a considerable element of researching cancer progression [17]. And the overexpression efficiency of CTNNB1 was measured by RT-qPCR and western blot assays in pcDNA3.1/CTNNB1 transfected DU145 cell (Fig. 5a). Then TOP/FOP flash assay suggested that pcDNA3.1/CTNNB1 or LiCl treatment offset the interceptive role of sh-LINC00689 in Wnt pathway. LINC00689 knockdown-mediated suppressed function on $\beta$-catenin activity was offset by CTNNB1 overexpression or $\mathrm{LiCl}$ treatment (Fig. 5b). RT-qPCR and western blot assays detected that mRNA and protein level of Wnt-related proteins (CTNNB1/ $\beta$-catenin, CCND1, CDK2 and c-MYC) were reduced by knocking down LINC00689, then this inhibitory effect was counteracted by CTNNB1 upregulation or $\mathrm{LiCl}$ supplement (Fig. 5c, d). In short, LINC00689 activates Wnt pathway via upregulating CTNNB1 in PCa.

\section{LINC00689 promotes prostate cancer progression by upregulating CTNNB1}

The rescuing role of miR-496 inhibitor in sh-LINC00689 transfected PCa cells has been validated, and whether LINC00689 regulated the development of PCa through CTNNB1 needed further investigation. Through MTT and colony formation assays, upregulation of CTNNB1 reversed the inhibiting effects of knocking down LINC00689 on cell proliferation (Fig. 6a, b). In a similar way, LINC00689 silencing spurred DU145 cell apoptosis, and this stimulating effect was partly offset by overexpressing CTNNB1. And for flow cytometry analysis results, in sh-LINC00689\#1 group, cell apoptosis rate had about two folds of increase compared with sh-NC group. In sh-LINC00689\#1+ pcDNA3.1/CTNNB1 group, cell apoptosis rate declined and decreased about two folds in comparison with sh-LINC00689\#1 group (Fig. 6c). Additionally, CTNNB1 overexpression neutralized the activating influence of LINC00689 downregulation on cleaved caspase 3 protein expression and caspase 3 activity (Fig. 6d, e and Additional file 1c). On the other hand, transwell assay proved the rescuing role of pcDNA3.1/CTNNB1 in DU145 cell migration and invasion restrained by LINC00689 insufficiency (Fig. 6f, g). In general, LINC00689 promotes PCa progression by upregulating CTNNB1 expression Additional file 1, 2.

\section{Discussion}

$\mathrm{PCa}$ is one of the most prevalent malignancies for men $[18,19]$. Additionally, the older man is, and the more likely he is to suffer from $\mathrm{PCa}$, and $70-80$ years old is the peak of having $\mathrm{PCa}$ [20]. Nonetheless, family hereditary PCa occurs when men are relatively young, and nearly half of patients with family hereditary $\mathrm{PCa}$ are under 56 [21]. The therapeutic methods of PCa include chemotherapy, radiation treatment and operation. Currently, proton therapy has no damage to non-tumor tissue and is a safe and effective way for treating PCa [22]. Nevertheless, the exact etiology and pathogenesis of $\mathrm{PCa}$ are poorly comprehended. Therefore, efforts are needed to explore more effective and specific measures to prevent PCa.

And previous studies have confirmed the role of lncRNAs in the initiation and development of diverse cancers, such as gastric cancer [23, 24], hepatocellular carcinoma $[25,26]$, renal carcinoma [27, 28], breast cancer [29, 30]. Regarding $\mathrm{PCa}$, there have been some related studies about the role of lncRNAs [31-33]. Nonetheless, the function of LINC00689 in PCa had not been illustrated yet. In our research, LINC00689 exhibited extremely high expression in PCa tissues and cells compared with control groups. Further, the expression of LINC00689 in patients with advanced $\mathrm{PCa}$ was higher than that in patients with early PCa. Meanwhile, the higher the expression of LINC00689, the shorter the overall survival time of patients with PCa. Subsequently, depletion

\footnotetext{
(See figure on next page.)

Fig. 6 LINC00689 promotes prostate cancer progression by overexpressing CTNNB1. a, b MTT (cultured for 0, 1, 2, 3, 4 days) and colony formation (after 14 days of incubation) assays detected DU145 cell proliferation in differently transfected groups (sh-NC, sh-LINC00689\#1 and sh-LINC00689\#1 + pcDNA3.1/CTNNB1). c Flow cytometry measured DU145 cell apoptosis in differently transfected groups (sh-NC, sh-LINC00689\#1 and sh-LINC00689\#1 + pCDNA3.1/CTNNB1). d Western blot assay measured cleaved caspase 3 expression in differently transfected groups (sh-NC, sh-LINC00689\#1 and sh-LINC00689\#1 + pCDNA3.1/CTNNB1). GAPDH was an internal control. e Caspase 3 activity was measured in DU145 cell proliferation in differently transfected groups (sh-NC, sh-LINC00689\#1 and sh-LINC00689\#1 + pcDNA3.1/CTNNB1). f, g Transwell assay (incubated for 24 h) measured DU145 cell migration and invasion in differently transfected groups (sh-NC, sh-LINC00689\#1 and sh-LINC00689\#1 + pcDNA3.1/ CTNNB1). Images were captured by using the inverted microscope $(4 \times$ objective lens) (scale bar $=100 \mu \mathrm{m})$. Error bars represent the mean \pm SD of at least three independent experiments. ${ }^{* *} \mathrm{P}<0.01$
} 


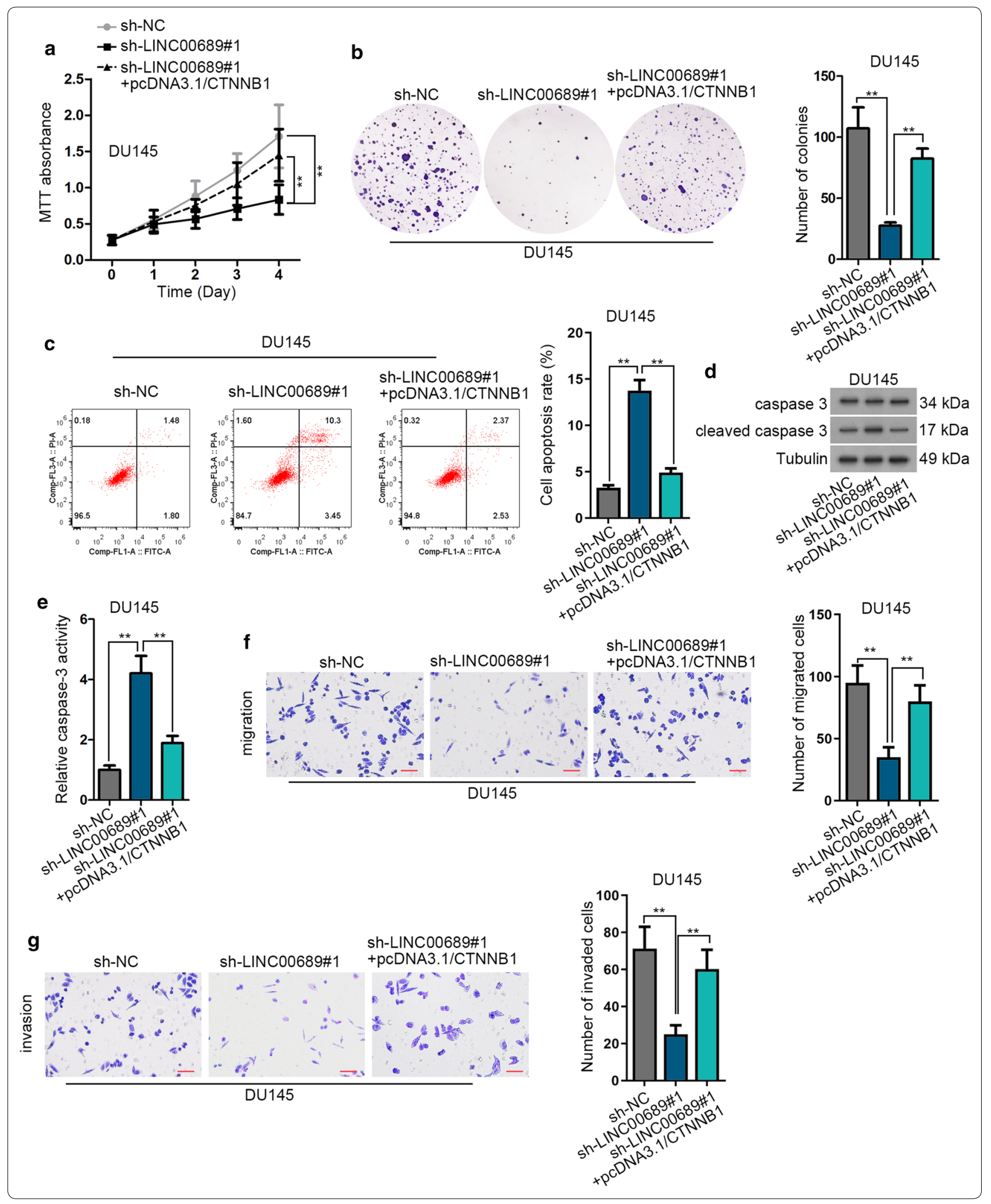


of LINC00689 inhibited PCa cell proliferation, migration and invasion, but promoted PCa cell apoptosis, indicating that LINC00689 served as an oncogene in PCa.

LncRNAs usually sponge with microRNAs (miRNAs) in cancers [34-36]. In this study, LINC00689 could bind with miR-496, and LINC00689 negatively modulated the expression of miR-496. In addition, the tumor suppressor role of miR-496 has been illustrated in non-small cell lung cancer [37], lung adenocarcinoma [38], bladder cancer [39], and osteosarcoma [40]. In the present study, miR-496 suppression could neutralize the inhibitory effects of LINC00689 silencing on PCa cell progression, suggesting the anti-tumor function of miR-496 in PCa. In other words, LINC00689 could promote the development of PCa via inhibiting miR-496.

Messenger RNAs (mRNAs) are indispensable members of ceRNA together with lncRNAs and miRNAs in cancer progression [41-43]. In our study, CTNNB1 acted as a target gene of miR-496, and the expression of CTNNB1 was separately negatively and positively regulated by miR-496 and LINC00689 in PCa. Furthermore, LINC00689 could regulate CTNNB1 via sponging miR496. In addition, Wnt is identified as an important pathway to regulate cell growth, apoptosis, migration and invasion [44-46]. In this study, LINC00689 knockdown inhibited Wnt pathway, and CTNNB1 upregulation rescued this restraining effect. In addition, overexpressing CTNNB1 counteracted the prohibitive impacts of LINC00689 insufficiency on cell proliferation, migration and invasion as well as the stimulating function of that on cell apoptosis.

\section{Conclusion}

In a summary, LINC00689 promotes PCa progression via regulating miR-496/CTNNB1 to activate Wnt pathway, which possibly bring about new idea for PCa treatment.

\section{Supplementary information}

Supplementary information accompanies this paper at https://doi. org/10.1186/s12935-020-01280-1.

Additional file 1. The untrimmed whole western blots.

Additional file 2. Specific steps of using starBase v3.0.

\section{Abbreviations}

ANOVA: Analysis of variance; ATCC: American type culture collection; BDNF-AS: Brain derived neurotrophic factor antisense RNA; CDNA: Complementary DNA; ceRNA: Competing endogenous RNA; DMSO: Dimethylsulfoxide; FBS: Fetal bovine serum; GAPDH: Glyceraldehyde-3-phosphate dehydrogenase: LINC00689: Long intergenic non-protein coding RNA 689; IncRNA: Long noncoding RNA; MALAT-1: Metastasis associated lung adenocarcinoma transcript 1; miRNA: microRNA; mRNA: Messenger RNA; MTT: Methyl thiazolyl tetrazolium; NC: Negative control; OD: Optical density; PBS: Phosphatebuffered saline; PCa: Prostate cancer; PVDF: Polyvinylidene fluoride; RIP: RNA immunoprecipitation; RISC: RNA-induced silencing complex; RT-qPCR:
Real-time quantitative polymerase chain reaction; SD: Standard deviation; SDS-PAGE: Sodium dodecyl sulfate polyacrylamide gel electrophoresis; shRNA: Short-hairpin RNA; SOCS2-AS1: Suppressor of cytokine signaling 2 antisense RNA 1; SPSS: Statistic package for social science; UTR: Untranslated region.

\section{Acknowledgements}

We appreciate the technical supports of laboratory members.

\section{Authors' contributions}

Project administration:LM. Data curation: ZL. Formal analysis: YC. Writing original draft: DL. Investigation: LM. Writing revised: ZL. All authors read and approved the final manuscript.

\section{Funding}

None.

\section{Availability of data and materials}

Not applicable.

\section{Ethics approval and consent to participate}

The present research was favored by the Ethics Committee of Affiliated Hospital of Jining Medical University. Informed consent was attained from all the patients.

\section{Consent for publication}

All authors have authorized the publication of this manuscript.

\section{Competing interests}

Authors declare no conflicts of interest in this study.

\section{Author details}

${ }^{1}$ Department of Urology, Qilu Hospital of Shandong University, No.107 Culture Road, Jinan 250000, Shandong, China. ${ }^{2}$ Department of Urology, Affiliated Hospital of Jining Medical University, Jining 272000, Shandong, China. ${ }^{3}$ School of Nursing, Shandong University, No.44 Culture Road, Jinan 250000, Shandong, China.

Received: 22 October 2019 Accepted: 18 May 2020

Published online: 05 June 2020

\section{References}

1. Kmetova Sivonova M, Jurecekova J, Tatarkova Z, Kaplan P, Lichardusova L, Hatok J. The role of CYP17A1 in prostate cancer development: structure, function, mechanism of action, genetic variations and its inhibition. Gen Physiol Biophys. 2017;36(5):487-99.

2. Chang J, Xu W, Liu G, Du X, Li X. Downregulation of Rab23 in prostate cancer inhibits tumor growth in vitro and in vivo. Oncol Res. 2017;25(2):241-8.

3. Chen F, Xiong W, Dou K, Ran Q. Knockdown of FOXK1 suppresses proliferation, migration, and invasion in prostate cancer cells. Oncol Res. 2017;25(8):1261-7.

4. Zhao Y, Chen W, Zhu W, Meng H, Chen J, Zhang J. Overexpression of interferon regulatory factor 7 (IRF7) reduces bone metastasis of prostate cancer cells in mice. Oncol Res. 2017;25(4):511-22.

5. Sun Y, Jin JG, Mi WY, Zhang SR, Meng Q, Zhang ST. Long noncoding RNA UCA1 targets miR-122 to promote proliferation, migration, and invasion of glioma cells. Oncol Res. 2018;26(1):103-10.

6. Meng YB, He X, Huang YF, Wu QN, Zhou YC, Hao DJ. Long noncoding RNA CRNDE promotes multiple myeloma cell growth by suppressing miR-451. Oncol Res. 2017;25(7):1207-14.

7. Maeda N, Kasukawa T, Oyama R, Gough J, Frith M, Engstrom PG, Lenhard B, Aturaliya RN, Batalov S, Beisel KW, et al. Transcript annotation in FANTOM3: mouse gene catalog based on physical cDNAs. PLoS Genet. 2006:2(4):e62.

8. Djebali S, Davis CA, Merkel A, Dobin A, Lassmann T, Mortazavi A, Tanzer A, Lagarde J, Lin W, Schlesinger F, et al. Landscape of transcription in human cells. Nature. 2012;489(7414):101-8.

9. Guttman M, Amit I, Garber M, French C, Lin MF, Feldser D, Huarte M, Zuk O, Carey BW, Cassady JP, et al. Chromatin signature reveals over a 
thousand highly conserved large non-coding RNAs in mammals. Nature. 2009;458(7235):223-7.

10. Batista PJ, Chang HY. Long noncoding RNAs: cellular address codes in development and disease. Cell. 2013;152(6):1298-307.

11. Misawa A, Takayama K, Urano T, Inoue S. Androgen-induced long noncoding RNA (InCRNA) SOCS2-AS1 promotes cell growth and inhibits apoptosis in prostate cancer cells. J Biol Chem. 2016;291(34):17861-80.

12. Ren S, Liu Y, Xu W, Sun Y, Lu J, Wang F, Wei M, Shen J, Hou J, Gao X, et al. Long noncoding RNA MALAT-1 is a new potential therapeutic target for castration resistant prostate cancer. J Urol. 2013;190(6):2278-87.

13. Li W, Dou Z, We S, Zhu Z, Pan D, Jia Z, Liu H, Wang X, Yu G. Long noncoding RNA BDNF-AS is associated with clinical outcomes and has functional role in human prostate cancer. Biomed Pharm. 2018;102:1105-10.

14. Liu X, Zhu Q, Guo Y, Xiao Z, Hu L, Xu Q. LncRNA LINC00689 promotes the growth, metastasis and glycolysis of glioma cells by targeting miR338-3p/PKM2 axis. Biomed Pharm. 2019;117:109069.

15. Jones DZ, Schmidt ML, Suman S, Hobbing KR, Barve SS, Gobejishvili L, Brock G, Klinge CM, Rai SN, Park J, et al. Micro-RNA-186-5p inhibition attenuates proliferation, anchorage independent growth and invasion in metastatic prostate cancer cells. BMC Cancer. 2018;18(1):421.

16. Pashaei E, Pashaei E, Ahmady M, Ozen M, Aydin N. Meta-analysis of miRNA expression profiles for prostate cancer recurrence following radical prostatectomy. PLoS ONE. 2017;12(6):e0179543.

17. Zhang LS, Lum L. Chemical modulation of WNT signaling in cancer. Prog Mol Biol Transl Sci. 2018;153:245-69.

18. Cai DW, Chen D, Sun SP, Liu ZJ, Liu F, Xian SZ, Wu PS, Kong GQ. Overexpression of PER3 reverses paclitaxel resistance of prostate cancer cells by inhibiting the Notch pathway. Eur Rev Med Pharm Sci. 2018;22(9):2572-9.

19. Feldman BJ, Feldman D. The development of androgen-independent prostate cancer. Nat Rev Cancer. 2001;1(1):34-45.

20. Thompson IM, Tangen CM. Prostate cancer screening comes of age. Lancet. 2014;384(9959):2004-6.

21. Ferlay J, Soerjomataram I, Dikshit R, Eser S, Mathers C, Rebelo M, Parkin DM, Forman D, Bray F. Cancer incidence and mortality worldwide: sources, methods and major patterns in GLOBOCAN 2012. Int J Cancer. 2015;136(5):E359-86.

22. Hanna C, Jones RJ. Emerging treatments for recurrent prostate cancer. Future oncology (London, England). 2015;11(21):2873-80.

23. Fu JW, Kong Y, Sun X. Long noncoding RNA NEAT1 is an unfavorable prognostic factor and regulates migration and invasion in gastric cancer. J Cancer Res Clin Oncol. 2016;142(7):1571-9.

24. Huang H, Liao W, Zhu X, Liu H, Cai L. Knockdown of long noncoding RNA GHET1 inhibits cell activation of gastric cancer. Biomed Pharm. 2017;92:562-8.

25. Gong J, Qi X, Zhang Y, Yu Y, Lin X, Li H, Hu Y. Long noncoding RNA linc00462 promotes hepatocellular carcinoma progression. Biomed Pharm. 2017:93:40-7.

26. Ge Z, Cheng Z, Yang X, Huo X, Wang N, Wang H, Wang C, Gu D, Zhao $\mathrm{F}$, Yao $\mathrm{M}$, et al. Long noncoding RNA SchLAH suppresses metastasis of hepatocellular carcinoma through interacting with fused in sarcoma. Cancer Sci. 2017;108(4):653-62.

27. Zhang M, Lu W, Huang Y, Shi J, Wu X, Zhang X, Jiang R, Cai Z, Wu S. Downregulation of the long noncoding RNA TUG1 inhibits the proliferation, migration, invasion and promotes apoptosis of renal cell carcinoma. J Mol Histol. 2016:47(4):421-8.

28. He X, Sun F, Guo F, Wang K, Gao Y, Feng Y, Song B, Li W, Li Y. Knockdown of long noncoding RNA FTX inhibits proliferation, migration, and invasion in renal cell carcinoma cells. Oncol Res. 2017;25(2):157-66.

29. Kim J, Piao HL, Kim BJ, Yao F, Han Z, Wang Y, Xiao Z, Siverly AN, Lawhon $\mathrm{SE}$, Ton BN, et al. Long noncoding RNA MALAT1 suppresses breast cancer metastasis. Nat Genet. 2018;50(12):1705-15.

30. Li H, Zhu L, Xu L, Qin K, Liu C, Yu Y, Su D, Wu K, Sheng Y. Long noncoding RNA linc00617 exhibits oncogenic activity in breast cancer. Mol Carcinog. 2017;56(1):3-17.
31. Zhou W, Tao Z, Wang Z, Hu W, Shen M, Zhou L, Wen Z, Yu Z, Wu X, Huang $K$, et al. Long noncoding RNA PCA3 gene promoter region is related to the risk of prostate cancer on Chinese males. Exp Mol Pathol. 2014;97(3):550-3.

32. Zhao R, Sun F, Bei X, Wang X, Zhu Y, Jiang C, Zhao F, Han B, Xia S. Upregulation of the long non-coding RNA FALEC promotes proliferation and migration of prostate cancer cell lines and predicts prognosis of PCa patients. Prostate. 2017;77(10):1107-17.

33. Li J, Zhang Z, Xiong L, Guo C, Jiang T, Zeng L, Li G, Wang J. SNHG1 IncRNA negatively regulates miR-199a-3p to enhance CDK7 expression and promote cell proliferation in prostate cancer. Biochem Biophys Res Commun. 2017:487(1):146-52

34. Peng W, Deng W, Zhang J, Pei G, Rong Q, Zhu S. Long noncoding RNA ANCR suppresses bone formation of periodontal ligament stem cells via sponging miRNA-758. Biochem Biophys Res Commun. 2018:503(2):815-21.

35. Chen X, Zhao X, Ma X, Gao G, Yu L, Yan D, Dong H, Sun W. Long noncoding RNA ILF3-AS1 promotes cell proliferation, migration, and invasion via negatively regulating miR-200b/a/429 in melanoma. Biosci Rep. 2017. https://doi.org/10.1042/BSR20171031.

36. Zhu C, Cheng D, Qiu X, Zhuang M, Liu Z. Long noncoding RNA SNHG16 promotes cell proliferation by sponging MicroRNA-205 and upregulating ZEB1 expression in osteosarcoma. Cell Physiol Biochem. 2018;51(1):429-40.

37. Ma R, Zhu P, Liu S, Gao B, Wang W. miR-496 suppress tumorigenesis via targeting BDNF-mediated PI3K/Akt signaling pathway in non-small cell lung cancer. Biochem Biophys Res Commun. 2019;518(2):273-7.

38. Lu QC, Rui ZH, Guo ZL, Xie W, Shan S, Ren T. LncRNA-DANCR contributes to lung adenocarcinoma progression by sponging miR-496 to modulate mTOR expression. J Cell Mol Med. 2018;22(3):1527-37.

39. Wu D, Zhang T, Wang J, Zhou J, Pan H, Qu P. Long noncoding RNA NNTAS1 enhances the malignant phenotype of bladder cancer by acting as a competing endogenous RNA on microRNA-496 thereby increasing HMGB1 expression. Aging. 2019;11(24):12624-40.

40. Qi NN, Tian S, Li X, Wang FL, Liu B. Up-regulation of microRNA-496 suppresses proliferation, invasion, migration and in vivo tumorigenicity of human osteosarcoma cells by targeting elF4E. Biochimie. 2019;163:1-11.

41. Lu G, Li Y, Ma Y, Lu J, Chen Y, Jiang Q, Qin Q, Zhao L, Huang Q, Luo Z, et al. Long noncoding RNA LINC00511 contributes to breast cancer tumourigenesis and stemness by inducing the miR-185-3p/E2F1/Nanog axis. J Exp Clin Cancer Res. 2018;37(1):289.

42. Wang $\mathrm{K}$, Jin W, Song Y, Fei X. LncRNA RP11-436H11.5, functioning as a competitive endogenous RNA, upregulates BCL-W expression by sponging miR-335-5p and promotes proliferation and invasion in renal cell carcinoma. Mol Cancer. 2017;16(1):166.

43. Sui CJ, Zhou YM, Shen WF, Dai BH, Lu JJ, Zhang MF, Yang JM. Long noncoding RNA GIHCG promotes hepatocellular carcinoma progression through epigenetically regulating miR-200b/a/429. J Mol Med. 2016;94(11):1281-96.

44. Ge YX, Wang CH, Hu FY, Pan LX, Min J, Niu KY, Zhang L, Li J, Xu T. New advances of TMEM88 in cancer initiation and progression, with special emphasis on Wnt signaling pathway. J Cell Physiol. 2018;233(1):79-87.

45. Yin P, Wang W, Zhang Z, Bai Y, Gao J, Zhao C. Wnt signaling in human and mouse breast cancer: focusing on Wnt ligands, receptors and antagonists. Cancer Sci. 2018;109(11):3368-75.

46. Dong X, Liao W, Zhang L, Tu X, Hu J, Chen T, Dai X, Xiong Y, Liang W, Ding $\mathrm{C}$, et al. RSPO2 suppresses colorectal cancer metastasis by counteracting the Wnt5a/Fzd7-driven noncanonical Wnt pathway. Cancer Lett. 2017;402:153-65

\section{Publisher's Note}

Springer Nature remains neutral with regard to jurisdictional claims in published maps and institutional affiliations. 